\title{
OPTIMAL ANNUAL ROUTINES: NEW TOOLS FOR CONSERVATION BIOLOGY?
}

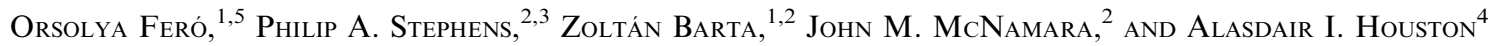 \\ ${ }^{1}$ Behavioural Ecology Research Group, Department of Evolutionary Zoology, University of Debrecen, H-4010 Debrecen, Hungary \\ ${ }^{2}$ Centre for Behavioural Biology, Department of Mathematics, University of Bristol, University Walk, \\ Bristol BS8 1TW United Kingdom \\ ${ }^{3}$ School of Biological and Biomedical Sciences, University of Durham, South Road, Durham DH1 3LE United Kingdom \\ ${ }^{4}$ Centre for Behavioural Biology, School of Biological Sciences, University of Bristol, Woodland Road, \\ Bristol BS8 1UG United Kingdom
}

\begin{abstract}
Many applied problems in ecology and conservation require prediction, and population models are important tools for that purpose. Formerly, the majority of predictive population models were based on matrix models. As the limitations of classical matrix models have become clearer, the use of individual-based models has increased. These models use behavioral rules imposed at the level of the individual to establish the emergent consequences of those rules at the population level. Individual behaviors in such models use an array of different rule types, from empirically derived probabilities to long-term fitness considerations. There has been surprisingly little discussion of the strengths and weaknesses of these different rule types. Here, we consider different strategies for modeling individual behaviors, together with some problems associated with individual-based models. We propose a novel approach based on modeling optimal annual routines. Annual routines allow individual behaviors to be predicted over a whole annual cycle within the context of long-term fitness considerations. Temporal trade-offs between different behaviors are automatically included in annual routine models, overcoming some of the primary limitations of other individual-based models. Furthermore, as well as population predictions, individual behaviors and indices of condition are emergent features of annual routine models. We show that these can be more sensitive to environmental change than population size, offering alternative, repeatable metrics for monitoring population status. Annual routine models provide no panacea for the problems of data limitations in predictive population modeling. However, as a result of their ability to deal with life-history trade-offs, as well as their potential for relatively rapid and accurate validation and parameterization, we suggest that annual routine models have strong potential for predictive population modeling in applied conservation settings.
\end{abstract}

Key words: environmental change; habitat degradation; individual-based models; migration; molt; optimality; population dynamics.

\section{INTRODUCTION}

Ecologists are often required to make predictions regarding the likely responses of populations to changes in their environment. Field trials, monitoring, experimental approaches, and expert opinion are important tools for making such predictions (Sutherland and Watkinson 2001, Sutherland 2006). Field trials, however, can usually be conducted only on restricted spatial or temporal scales, and short-term outcomes may not be borne out over longer periods (e.g., Freckleton et al. 2004). Monitoring can yield "natural experiments" that provide valuable information on the responses of populations to change (e.g., Gosler et al. 1995). However, such opportunities are rare and will often be confounded by changes in factors other than the

Manuscript received 21 June 2007; revised 14 February 2008; accepted 21 February 2008. Corresponding Editor: D. F. Parkhurst.

${ }^{5}$ E-mail: feroo@vocs.unideb.hu environmental variable of interest. Furthermore, predictions based on the results of such monitoring will often be unreliable if extrapolated beyond the range of situations previously encountered (Goss-Custard and Sutherland 1997, Coulson et al. 2001, Stephens et al. 2003). Expert opinion underlies many decisions in applied ecology (White et al. 2005) but is seldom based on structured, rigorous approaches and reinforced by subsequent evaluation (Sutherland 2006). Owing to the unreplicated nature of global change, therefore, and as a result of the weaknesses of alternative approaches, it has long been clear that field and experimental methods must be supplemented by predictive modeling (Shugart et al. 1992).

Predictive population modeling is an extremely varied enterprise, with few authors agreeing on the attributes and approaches to modeling that are necessary for making useful predictions. Perhaps the most common application of predictive population models is population viability analysis (PVA; Shaffer 1981, Gilpin and Soulé 1986, Boyce 1992, Akçakaya et al. 2004). Models 
constructed for this purpose tend to be matrix models, in which the population is classified by age or stage, and parameters are estimated that describe the probabilities of transitions between those stages (e.g., Caswell 2001). There has been considerable debate about the value of PVA (Boyce 1992, Hamilton and Moller 1995, Beissinger and Westphal 1998, Reed et al. 1998, Bierzychudek 1999, Brook et al. 2000, 2002, Coulson et al. 2001, Ellner et al. 2002). In particular, there is increasing recognition that traditional, age- or stage-based matrix models are limited in their ability to accommodate important detail at the level of individuals (Huston et al. 1988, Judson 1994, DeAngelis and Mooij 2005). Moreover, for predicting the consequences of novel conditions, it is also clear that mechanistic processes should be incorporated in population models (Sutherland 1996, 1998, Łomnicki 1999, Bradbury et al. 2001, Norris and Stillman 2002, Stephens et al. 2002a, b, 2003, Sutherland and Norris 2002).

As a result of growing awareness of the limitations of matrix modeling for incorporating many processes of interest, both individual-based models and behaviorbased models are becoming more widespread (DeAngelis and Mooij 2005, Sutherland 2006). These models focus on parameterizing the actions of individuals, such that the characteristics and trajectories of populations are emergent properties of simple rules imposed at the individual level. No easy definition of an individualbased model exists (Grimm and Railsback 2005). The distinctions between individual-based models and behavior-based models are mainly historical; the two approaches seem to have developed from different sets of underlying rules, in parallel literatures with woefully little integration. Hereafter, we refer to these modeling approaches, collectively, as individual-based models (IBMs; though behavior-based models, BBMs, are distinguished as a special case where necessary).

There have been several recent reviews of the current use of IBMs (Grimm 1999, Norris and Stillman 2002, DeAngelis and Mooij 2005, Sutherland 2006). DeAngelis and Mooij (2005), in particular, provide a thorough classification of the range of purposes to which IBMs have been put, while Sutherland (2006) details the applications of BBMs, as well as the ways in which they have been tested. In spite of these reviews, detailed assessments of the mechanisms underlying such models are lacking. The mechanisms of interest to us here are those that encapsulate the individual processes of "Adaptation," "Fitness," "Sensing," and "Prediction" (sensu Grimm et al. 2006).

Our aim is to consider the problems associated with available methods used in IBMs and to suggest a possible alternative. First, we assess the type of rules that are imposed at the individual level in IBMs. These range from probabilistic rules to detailed, game-theoretic optimization procedures. We discuss the potential applications and drawbacks of each approach. Second, we give a brief overview of some other problems associated with IBMs. Third, we suggest an alternative approach, modeling optimal annual routines (OARs) that might overcome some (though not all) of those problems. We consider the advantages of OAR models and the situations in which they might be useful. We also give an example to illustrate the ways in which OARs could be used in predictive, conservation settings. We conclude with a summary of remaining problems and opportunities for individual-based predictive modeling.

\section{IBMs, Rules, AND Optimality}

Following DeAngelis and Mooij (2005), we consider individual-based models (IBMs) to be simulation models in which the fates of "agents" (typically representing individuals) can be followed according to the unique attributes of those agents. The behavior (and, thus, the fate) of agents is contingent on their history of interactions with the environment and other agents, as well as aspects of their current situation. Beyond this, it is difficult to generalize about IBMs, because the behaviors of interest will vary according to the application of the model. For example, if the model is aimed at assessing the consequences of assortative mating (e.g., Sadedin and Littlejohn 2003), the key behaviors might include matechoice and mate-search rules. By contrast, if the purpose of the model is to assess habitat use (e.g., Sutherland and Allport 1994), the key behavior might involve movement to maximize intake rate. In all cases, however, the behaviors will have consequences at each time step, typically manifested in stochastic changes in the attributes of the agents, as well as either stochastic or deterministic changes in their environment.

The key point to notice is that all IBMs depend on agents behaving in a manner contingent on their attributes. There is, however, wide variation in the rules used to determine those behaviors. We recognize two main categories of rule: empirically derived rules and optimality rules. The first category usually derives from direct observation, while those in the second category (including either short-term proxies for optimality or long-term fitness considerations) typically derive from theoretical considerations. These categories need not be completely exclusive. Stephens and Krebs (1986: Chapter 8) noted that there are many examples of behaviors that follow simple rules, rather than rules informed by long-term fitness considerations. Indeed, simple rules can perform well and may represent optimal strategies if more complex rules lead to trade-offs, owing to their demand for investment in sensory apparatus or knowledge (McNamara and Houston 1980, Houston et al. 1982, Stephens and Krebs 1986). Our distinction between simple, empirically derived rules and optimality rules is thus based on modeling philosophy rather than any fundamental view of the information on which animals base their actions. The difference between IBMs in general, and BBMs as a special case, might be related to their different origins on the spectrum of rule types. Specifically, it appears that IBMs began with empirically 
derived rules, while BBMs originated among modelers who were concerned with the optimality end of the spectrum. Increasingly, however, the distinction is blurred, and many models contain behavioral processes determined by a combination of rule types. We now discuss these different rule types and their major attributes.

\section{Empirically derived rules}

Empirically derived rules are typically based on estimates of the probability with which an individual in the current state would perform one of a set of possible actions. As an example, consider the model of Grimm et al. (2003) concerning persistence in populations of the alpine marmot Marmota marmota. This is a spatially explicit model, with individuals classified by sex, age, group, and social status. A key behavior determining the persistence of a social, group-living species like the alpine marmot is subordinate dispersal. Dispersal determines group size (a critical determinant of overwinter survival in marmots; Grimm et al. 2003), as well as the rate at which existing groups die out and vacant territories are recolonized. In the model of Grimm et al. (2003), dispersal (or remaining in the natal territory) is a stochastic process, with age-specific probabilities taken directly from empirical data.

IBMs based on probabilistic rules are similar to matrix models in that transition probabilities are, as with most matrix models, taken from empirical data. The inclusion of spatial, social, or kin relationships usually requires that the models are individual based, but behaviors are consistent for large sections of the population, regardless of the specifics of an individual's situation. One problem with this approach was identified by Stephens et al. (2002a). These authors compared a spatially explicit, probabilistic population model with a model in which individual decisions were context dependent (rather than fixed for individuals of given age and status). Stephens et al. (2002a) showed that predicted population growth was typically higher in the context-dependent model because subordinates were less likely to disperse from smaller groups (where their presence had a strong effect on the survival of related juveniles) and more likely to disperse from larger groups (where they had a lower effect on expected survival). These results were observed in spite of similar levels of age-specific dispersal in both models and suggested that ignoring realistic patterns of behavior could lead to erroneous predictions (Stephens et al. 2002a).

Another problem with probabilistic behavioral rules has already been alluded to: empirical probabilities determined in one set of conditions cannot be expected to apply in novel conditions. In the case of the alpine marmot models, consider the consequences of a declining population. A small population might be composed of a few large groups (with a similar distribution of group sizes to that observed in the empirical study from which data were drawn). In that case, age-specific dispersal probabilities might be expected to be the same as for the field study or even higher (given the likelihood of increased territory availability). Alternatively, a small population might consist of many smaller groups. In this case, the contribution of each subordinate to the success of its group would be much higher, while territory availability would remain relatively restricted. Here, we would expect dispersal probabilities to be reduced. In either case, models based on probability lack any mechanistic underpinning for re-deriving the relevant probabilities. In the language of Grimm et al. (2006), they do not enable individual behavior to adapt to the individual's changing internal and external environment.

A third problem with empirically derived rules can be that they implicitly assume the form of some decisions. One example is the assumption that, if they are able to do so (i.e., if they have a nest site or territory and access to a mate), all reproductively mature individuals will attempt to breed. This may be broadly consistent with our interpretation of field observations. Nevertheless, theoretical ecology tells us that there is a trade-off between current and future reproduction; in adverse conditions, individuals may forego reproduction, rather than compromising survival (and, thus, future reproductive success; Stearns 1992, McNamara and Houston 1996).

In general, empirically derived rules do not allow for decisions that are contingent on their probable outcomes and, thus, ignore many of the insights that have emerged from theoretical ecology. Measured probabilities might have good heuristic value for exposing processes underlying the observed dynamics of populations, but are seldom adequate as rules governing key behaviors in predictive population models. In spite of this, most predictive IBMs must contain some empirically derived rules. To clarify that they are not unique to an isolated set of circumstances, probabilities should, ideally, be well supported by data from a range of circumstances. Failing that, consideration should be given to the likely consequences, and tests of model sensitivity to a range of plausible values should usually be conducted.

\section{Optimality rules}

Optimality-based approaches tend to form the core of models referred to as BBMs (Norris and Stillman 2002, Sutherland 2006). Optimality rules overcome many of the limitations of simpler decision rules but introduce a range of novel concerns. Sutherland (2006) noted that BBMs tend to focus on feedback mechanisms between density and population distribution or performance. The four principal mechanisms that have been studied are depletion, interference, territoriality, and social rank. Behavioral feedbacks can be predicted on the basis of short-term proxies for fitness (e.g., maximizing intake rate) or long-term fitness considerations (e.g., maximizing reproductive success).

Population models employing short-term proxies for fitness are usually based on the ideal free distribution 
(Fretwell and Lucas 1970), which assumes that individuals have an ideal knowledge of their options and are free to move about their habitat in order to maximize their fitness. Thus, behavior in these models is derived from maximizing the fitness payoff from a set of available options. These models are usually game theoretic in that the payoffs from any action depend on the actions of others. The vast majority of existing examples have focused on depletion and interference (e.g., Pettifor et al. 2000, Stillman et al. 2000, 2003, Gill et al. 2001). Both of these processes affect spatial and temporal variation in expected food intake, and so can predict distribution (Gill et al. 2001), mortality (Stillman et al. 2000, 2003), and seasonal switches between different resources (Pettifor et al. 2000).

Owing to the central importance of food intake rate in depletion and interference models, intake rate is usually the optimization criterion used to inform behavioral decisions (such as where to feed). We designate this a short-term proxy because it depends on the assumption that maximizing intake rate in the short term will also maximize fitness in the longer term. When models focus on stages of the life cycle in which survival can only be achieved by maintaining a high intake rate, this assumption may be justified. However, there are two problems with this approach. First, even when considering times of year when animals are food stressed, maximizing intake may not equate to maximizing survival (and thus long-term reproductive success; Houston and McNamara 1997). In addition, foraging is only one of several activities to which an individual can allocate time. For both of these reasons, animals face trade-offs between different elements of fitness (for example, trade-offs between the dangers of starvation and predation; McNamara and Houston 1987, Witter and Cuthill 1993, Houston and McNamara 1999), and abundant evidence suggests that, even at times of high energy demand, individuals regulate their intake below what is achievable (e.g., Rogers et al. 1994, Witter et al. 1995, Fauchald et al. 2004). Consequently, while shortterm proxies for fitness might be adequate in some situations, in general, the fitness consequences of actions can only be viewed in light of their long-term effects on an individual's lifetime reproductive success (Houston and McNamara 1999).

A smaller number of BBMs have attempted to use long-term, inclusive fitness measures to determine behavioral (typically dispersal) decisions (e.g., Stephens et al. 2002a, Ridley et al. 2003). In both of these examples, estimates of fitness were derived by forward projection, a process entailing a number of restrictive assumptions. For example, in the model of Ridley et al. (2003) of the Seychelles Warbler, Acrocephalus sechellensis, methods of estimating long-term fitness required the assumption that current conditions are indicative of future conditions (i.e., that an individual chooses to disperse by comparing estimated lifetime reproductive success if it stays in a group that is always of size $x$, to lifetime reproductive success if it departs for a group that is always of size $x-1$ ). In addition, methods for fitness estimation also meant that while the authors could consider the population processes operating within stable populations (in saturated habitats), it was not possible to predict population dynamics in unsaturated environments (Ridley et al. 2003).

The approach used by Stephens et al. (2002a) imposed similarly problematic restrictions. In particular, it was necessary to assume that successful dispersers would become dominant in groups of average size and composition, in spite of the implications that resultant behaviors would have for the size and composition of groups. Similarly, it was also necessary to assume that the choice faced by a potential disperser was the choice between dispersing now or remaining in the natal territory and dispersing the following year (unless dominance was inherited in the meantime; Stephens et al. 2002a). This overlooks the breadth of choices faced by a potential disperser who can remain in the natal territory for any number of years before either dispersing or inheriting dominance.

In general, long-term fitness considerations are important for determining the consequences of current actions and, thus, for determining optimal behaviors. However, estimating fitness by forward projection is seldom satisfactory, partly because of the feedbacks between estimates of fitness and future conditions, but also because of the difficulties of incorporating future choices into current projections. These problems strongly suggest that a dynamic approach to estimating long-term fitness will yield marked improvements in the application of optimality rules to predictive population modeling.

\section{Problems with IBMs}

In the previous section we considered some of the problems associated specifically with empirically derived behavioral rules in IBMs. We noted that probabilistic rules are seldom appropriate for predictive population modeling, although they will usually be a necessary evil. We also observed that optimality approaches overcome many of the limitations of simpler decision rules but introduce a range of novel concerns. IBMs are subject to many of the more general concerns of predictive modeling (e.g., Boyce 1992:496-498). However, here we focus on a number of additional problems that are more strongly associated with IBMs that employ optimality rules. Optimality has, itself, been the subject of some debate (Pierce and Ollason 1987, Stearns and Schmid-Hempel 1987), but it is now generally recognized that optimality provides a useful basis for obtaining predictions regarding behavior (e.g., Sutherland 1996). Here we focus on concerns associated with the use of optimality rules in population modeling. We stress the drawbacks of BBMs in the context of models with which we are familiar but note that most of the problems to which we allude are widespread. In particular, we focus on the following: difficulties of 
parameterization, the problem of unknowns, and time horizons and prediction.

\section{Difficulties of parameterization}

Estimating the fitness consequences of current actions requires a detailed understanding of interactions both between individuals and between an individual and its environment. We have already discussed the complexities of estimating fitness in a long-term context, but even short-term proxies for fitness can be surprisingly difficult to parameterize. As an example, consider models that use food-intake rate as their criterion for optimization. Food intake typically depends on measures of food availability (which can be dependent on depletion) and the functional response (which relates food intake to availability and can be modified to account for intraspecific interference; Sutherland 1996). Stephens et al. (2003) noted that estimates of the parameters of the functional response from single species can show substantial variation, leading to concerns over the accuracy of resultant foraging models. Although emerging evidence suggests that, for some groups at least, functional responses may be predictable on the basis of readily measured characteristics (Piersma et al. 1995, GossCustard et al. 1996, Stillman and Simmons 2006), it remains to be seen whether this approach will be broadly applicable to a wide range of species.

Another problem associated with foraging models is that they require that the dynamics of the resource population are also well-understood (Stephens et al. 2003). For specialists feeding on one or a very few types of prey, this may present few problems, especially during periods when rates of resource renewal are low. However, for species with more varied diets, or at times when resources are growing or reproducing, understanding resource dynamics can be highly problematic.

Generally, difficulties with parameterizing optimalitybased IBMs arise because of the volume of detailed data required to estimate the consequences of individual decisions with confidence. As an example, to assess the fitness consequences of dispersal decisions in female meerkats, Suricata suricatta, Stephens et al. (2005) used a data set comprising over 240000 meerkat days of the observation of known individuals. In spite of the richness of this data set, the fates of only 20 dispersing females were known with conviction. Similarly, for their model of alpine marmots, Stephens et al. (2002a) used data from an intensive, 13-year field study. Again, however, data were available on the fates of only 22 long-distance dispersers and seven overwintering nonterritorial animals. Both of these examples illustrate well the difficulties of estimating the fitness consequences of dispersal, and more generally, the data-hungry nature of optimality rules in IBMs.

\section{Unknown parameters}

An extreme version of the parameterization problem arises with some parameters that simply cannot be measured. When estimating these parameters is the purpose of the model (e.g., Stillman et al. 2002), this need not be a problem. However, many models necessarily include unknowns, even where the models serve a much broader purpose than estimating those values. An example is the model of Stephens et al. (2002a) discussed earlier. In that model, it was necessary to estimate the future fitness of a subordinate that dispersed from its natal territory. Subordinate marmots cannot join an existing group as a subordinate, and in order to gain direct reproductive success, must establish their own group in a vacant territory or evict the current dominant from an occupied territory (Arnold 1990a, b). Clearly, empirical data can be used to determine the rate at which tenured dominants are evicted. However, the observed rate of eviction is an outcome of the type of animals dispersing, the rate of attempted evictions, and the probability of eviction. Thus, using an observed value for such a process is erroneous. Similar arguments have been advanced for problems with estimating the risk of predation (Lank and Ydenberg 2003). Many other important elements of IBMs are vulnerable to the same concerns, including factors such as the strength of interference, frequencies of disturbance, and unconsidered sources of mortality and predation (which can obviously change in either a compensatory or additive manner).

The key point is not that models that rely on unknown parameters should be avoided, but rather that the process of determining plausible values for unknown parameters often involves varying those parameters through a realistic range until a good model fit is achieved. Given that the fit of the model is usually an indicator of its quality, this is a circular process that can inflate the apparent success of a model. Ideally, available data sets would be divided into portions used for model specification and portions used for model validation. However, given the problems of parameterization alluded to earlier, this will often place unrealistic burdens on the available data. In these cases, it is essential that the process of estimating unknown parameters is acknowledged overtly and that the consequences for model fit are also recognized. Sensitivity analyses in these circumstances can identify to which of the unknown parameters model outcomes are most sensitive. This can suggest the most appropriate focus for empirical and analytical parameter estimation.

\section{Time horizons and prediction}

Perhaps owing to the complexities of parameterizing behavioral models of the breeding season on the basis of short-term proxies, few BBMs have been constructed that consider the full annual cycle (most focus only on the nonbreeding period; Sutherland 2006). Exceptions, such as the model of Stillman et al. (2001), tend to model the breeding period on the basis of empirical data on reproductive success, omitting any adaptive components of the model for this period. This poses two problems. 
First, where phenomenology must be invoked to describe the dynamics of populations during the breeding period, these models are subject to the concerns associated with models based on probabilistic rules. In particular, phenomenological models are constrained to making predictions for scenarios within the span of past observation (Stephens et al. 2003).

For models that focus only on the nonbreeding period, a second problem is encountered, whereby predictions of population level effects may not be borne out over the full annual cycle. For example, even where models predict a decrease in the number of individuals that emerge from winter, it is impossible to predict the effects of this decrease on the long-term dynamics of the population. Specifically, if the population is limited by competition for food during the breeding season, reduced breeding populations might increase fecundity, thus having little effect on long-term population size. For many species, there is evidence that population regulation is imposed most strongly outside the breeding season (e.g., Peach et al. 1999, Hole et al. 2002, Newton 2004, 2006), and for these, it might be possible to extrapolate from the implications of reductions in nonbreeding populations. In general, however, it cannot be assumed that specific periods can be considered in isolation (McNamara and Houston 2008). Rather, population dynamics must be viewed within the context of the entire annual routine.

\section{A Possible Alternative}

\section{Optimal annual routine models}

Recently, optimal annual routine models (McNamara et al. 1998, 2004, Houston and McNamara 1999, Barta et al. 2006a, b, 2008, McNamara and Houston 2008; McNamara et al., in press) have been developed to understand how annual periodicity affects optimal schedules of behavior within an annual cycle. We suggest that these models have great potential for predictive population modeling, and in this section, we outline why. We give an overview of optimal annual routines (OARs), highlighting why they are especially suitable for understanding and describing population processes. We also show that OARs overcome a number of the problems of other population models based on optimality rules (as just discussed).

OARs enable the identification of optimal behavioral strategies of individuals at each time step over an annual cycle in a periodically changing environment. The optimal behavior depends on the individual's attributes and the environment, and is determined by comparing the long-term fitness consequences of possible behavioral decisions at each time step. More precisely, OAR models find the optimal behavior by maximizing the number of descendants left far in the future, immediately providing an estimate of the survival and reproductive success of individuals that behave optimally. Thus, these models are ideal for describing population processes based on individual behavior. Having established state- dependent OARs, Monte Carlo simulations of optimally behaving individuals can be used to examine the behavior of individuals and the fate of whole populations.

In order to model population processes as the result of individual behavior, it is necessary to consider that carryover effects and cross-seasonal interactions can affect population trends considerably (Marra et al. 1998, Norris 2005, Webster and Marra 2005). It is important, therefore, to be able to describe the behavior of individuals over the longer term by considering the full annual cycle. For a number of reasons, this is not a simple task. First, to predict behavior over the full annual cycle, seasonal changes in the environment that affect individuals must be incorporated. Owing to periodicity, the value (the consequences for fitness) of actions performed (e.g., breeding, molt) can vary over the year (e.g., the survival and subsequent reproduction of young may depend on the time in the season at which they are born; Lack 1968, Green and Rothstein 1993, Szekely and Cuthill 1999, Cote and Festa-Bianchet 2001). Second, when modeling behavior over a long time, optimal behavior can only be determined reliably if the fitness consequences of possible future choices are incorporated. Performing one action can exclude the performance of other actions (for example, birds cannot breed while migrating) and affects the individual's future state, also influencing actions available in the future. Consequently, the optimal timing of an activity depends not only on the value of that action (which changes over time), but also on whether there are other appropriate times to perform the excluded activities (Foster 1975, Houston and McNamara 1999).

As we have discussed, most IBMs use forward projection and, consequently, have limited ability to deal with the kinds of future complexity necessary for predicting behaviors throughout an annual time frame. By contrast, OAR models are designed to overcome those very limitations. Importantly, game theory (e.g., Maynard Smith 1982) can easily be accommodated in OARs. Consequently, feedbacks between individuals, such as density dependence, can readily be included in models. OAR models are holistic in the sense that the possible actions are handled simultaneously; for example, breeding does not exclude molt a priori, but exclusion may emerge during the solution of the model. In general, these models do not use preliminary assumptions or empirical probabilities to determine any of the behavioral decisions. The optimal strategy is always found by calculating and comparing the longterm fitness consequences of the available sets of actions.

Technically, OARs are based on stochastic dynamic programming (Houston et al. 1988, Mangel and Clark 1988, Houston and McNamara 1999, Clark and Mangel 2000), incorporating the effects of various factors through state variables. This technique is used increasingly to determine and understand various behavioral choices, including optimal timing (e.g., McNamara et al. 
1998, Barta et al. 2008) and allocation patterns (e.g., Bunnell and Marschall 2003, Garvey and Marschall 2003; for an example involving conservation management decisions see Doherty et al. [1999]). In statedependent dynamic programming models it is not necessary to specify a priori the consequences for fitness of performing a given action; it is sufficient to describe only the effects of the actions on state variables, so that the fitness consequences of the different actions arise through the solution of the model. Also, it is possible to model trade-offs easily since, in general, they are manifested through the state variables (McNamara and Houston 1996). This enables details of physiology to be incorporated in the model (e.g., McNamara and Houston 2008).

OAR models involve two stages: first, optimal behavioral strategies for each state and each time of year are determined by backward iteration; next, forward stochastic simulations are run to assess the consequences of those optimal strategies. In the stochastic simulations, individuals use the state-dependent optimal behavioral strategies determined in the previous stage. Under this fitness-maximizing strategy, an individual's behavior depends only on its internal state (e.g., energy level) and some perceptible external circumstances (e.g., time of year). Additionally, errors in decision making can readily be included in OARs (McNamara et al. 1997), potentially allowing for departures from the assumption of perfection among modeled individuals.

\section{Advantages and disadvantages of OAR models}

The applicability of a model (or group of models) depends on how easily, simply, and reliably the model can be parameterized (providing the model with initial data), the validity of its predictions tested, and its predictions used. In general, OAR models are subject to many of the difficulties of parameterization to which optimality-based IBMs are subject. Though general implications can be produced relatively easily, the application of these models to real systems will require large data sets to describe the relationships and constraints and to estimate parameters. However, in terms of validation, optimal annual routine models have a significant advantage. Whereas most IBMs generate predictions at the population level, annual routines produce many individual-level indicators. These emerge because, in addition to predicting the fate and characteristics of the population, they also predict individual behavior (e.g., foraging intensity, timing of breeding) and state variables (e.g., level of energy reserves, immunocompetence). This can make model validation much more efficient because individual traits can usually be measured in a short period of time, whereas the estimation of attributes of populations requires data from a longer time period. Individual traits can also be measured more precisely (with less error) because, in this case, individuals (rather than populations) are the sampling units, so it is easier to increase both sample size and accuracy. Another significant advantage for model testing is that, since OAR models are dynamic, it is possible to predict various population parameters (e.g., age or size distributions, proportion of breeding individuals) at any point in time, and these predictions can also be tested by sampling over relatively short time frames. This also applies to the state variables (e.g., level of energy reserves, immunocompetence). Further work is needed, however, to clarify how the propagation of errors in these short-term estimates influences the precision of the predictions of the OAR models.

The approach of OARs also provides new possibilities regarding the use of model predictions. It is possible not only to simulate the effects of various endangering factors and the consequences of conservation measures on population dynamics, but also to predict individual behavior and the state variables in a changing environment. With the aid of simulations, we can examine whether the behavior or state variables respond more rapidly than population size to various environmental changes (Matsinos et al. 2002). For example, deteriorating environmental conditions might significantly decrease the immunocompetence of individuals well before the population starts to decline. This presents the possibility that OAR models could highlight early warning signals at an individual level that would prompt management actions before population-level signals could be detected. OAR models also enable examination of the response of behavior and state variables to changes in various environmental factors. Some variables may be more sensitive than others to certain environmental factors. If this is the case, monitoring the behavior and state variables of individuals will supply valuable information not only about the population trends but about their causes as well (Hill 1995, Lewis et al. 2006).

Though we put equal emphasis on behavior and state variables in the preceding discussion, practical considerations may dictate that state variables have a more important role. Collecting samples of behavior is usually more complex and takes more time. In some case, it may be relatively easy to measure individual state. For example, it is possible to estimate certain components of an individual's immunocompetence even from a single drop of blood (Pap 2002), and noninvasive methods for obtaining such samples are beginning to emerge (Becker et al. 2006). Consequently, monitoring populations based on state variables could serve as a new, sensitive tool for conservation biology.

One practical limitation of OAR models is that they are often complex, so model development and verification is likely to be slow and need expertise. Furthermore, solving these models requires long computations, even for modestly complicated cases, and computation time increases exponentially with the number of state variables. Therefore it is important to include only those state variables that are essential for the problem at hand and this requires substantial knowledge of the species 
under investigation. Nevertheless, with the power of a good desktop computer, reasonably complex models (with 4-6 state variables) can be solved within a day.

\section{Examples: Molt and Migration in Birds}

In this section, we present two case studies to illustrate the approach we advocate. We begin with a relatively simple example, considering molt strategies of nonmigratory birds. We then show how this relatively simple model can be extended to more complex situations (e.g., by including migration). Our intent is not to suggest that these particular examples have clear implications for conservation but, rather, to show the types of inputs and outputs that might be expected to have uses in a conservation setting.

\section{Optimal molt strategy of nonmigratory birds}

Barta et al. (2006b) developed an annual routine model to examine the optimal molt strategy of nonmigratory birds. In this model, individuals are characterized by five state variables: quality of (1) outer and (2) inner primaries (primaries are the 10 outermost flight feathers of the wings), (3) energy reserves, (4) breeding status, and (5) foraging experience. Quality of primaries influences flying efficiency: more energy is required to fly and predation risk increases as feather wear increases. Primaries degrade gradually, with the rate of degradation increasing with the bird's foraging intensity. Energy reserves increase during foraging and decrease because of the energetic costs of the activities performed. Breeding status specifies whether or not there are dependent young, and their age if they are present and being cared for. Experience, and hence foraging ability, of young increases with age. At the beginning of every week, a modeled bird selects its behavior for that week in terms of the following four classes of behavioral actions: (1) molt of the outer or (2) the inner primaries, (3) foraging intensity, and (4) reproductive behavior (starting to breed, caring for the brood, or abandoning it). The birds "live" in a seasonal environment where the amount of food varies sinusoidally over the year. There is explicit density dependence in the model, so its effect on the food supply is considered when determining the optimal strategy. Correspondingly, in the simulations, population size affects amount of food that is actually available to the individuals.

We used the optimal strategy (Fig. 1a) determined by Barta et al. (2006b) to run Monte Carlo simulations. In these simulations, the population of modeled individuals lived for 30 years under the conditions for which optimal strategies had been derived. After the 30th year, we gradually decreased the amount of food available to the population over certain periods of the year by $0.5 \%$ of the original value per year. In this example, we assume that the environment changes much more rapidly than the annual routine can adapt. To reflect this assumption, the optimal annual routine is not recalculated during the simulation (for more on this assumption see the Discussion). Note, however, that although the animals do not update the optimum behaviors associated with any given state, their behavior will change as a result of changes in their state.

Unsurprisingly, the food reduction led to a decline in the population size (Fig. 1b). However, we found that changes in levels of energy reserves followed a distinct pattern: food reduction at a given time had no effect on mean reserves measured during certain periods of the year, but when measured during other periods, significant increases in the energy reserves of individuals could be detected (Fig. 1c). Representing the changes of energy reserves and population size over years (Fig. 1d), it is clear that energy reserves started to respond to the food reduction much earlier than population size, illustrating the usefulness of state variables in studies of population processes.

\section{Optimal molt and migration strategies of birds}

Barta et al. (2008) extended the previous model to examine the optimal timing of molt and migration in the life cycle of migratory birds. To do this, a new state variable, location, was introduced. In the model, birds could choose between four locations with independent food distributions, a scenario that might represent a bird population migrating from Scandinavia to Africa, using two stopover sites along their route. To make movements between locations possible, a new behavioral action, migration, was also introduced. In the model, movement between neighboring locations took one week, so the whole migration lasted for at least three weeks. Barta et al. (2008) showed that the food distribution at each location plays an important role in the development of the two most common migrationmolt strategies of birds. If there is an abundant food peak on the breeding site in summer, then the birds molt immediately after breeding, before the autumn migration ("summer molt"). However, if the intense but short food peak in summer on the breeding site is followed by a high food peak in winter on the wintering site, then birds follow the so-called "winter molt" strategy, beginning their autumn journey immediately after breeding and molting only after arrival at the wintering site. We used this model to determine how a reduction of available food at migratory stopover sites is likely to affect the population dynamics of migratory birds. Monte Carlo simulations were run with the original food distributions for 30 years, after which the amount of available food was decreased (by $0.5 \%$ per year) on certain sites (the breeding site, the stopovers, or the wintering site), for populations following either the summer or the winter molt strategy. The effect of manipulations was measured by the time taken for the population size to halve. A small (3-7\%) reduction in food was sufficient to halve the populations (Fig. 2). Populations following the two strategies responded differently to the food reduction; however, in general, 

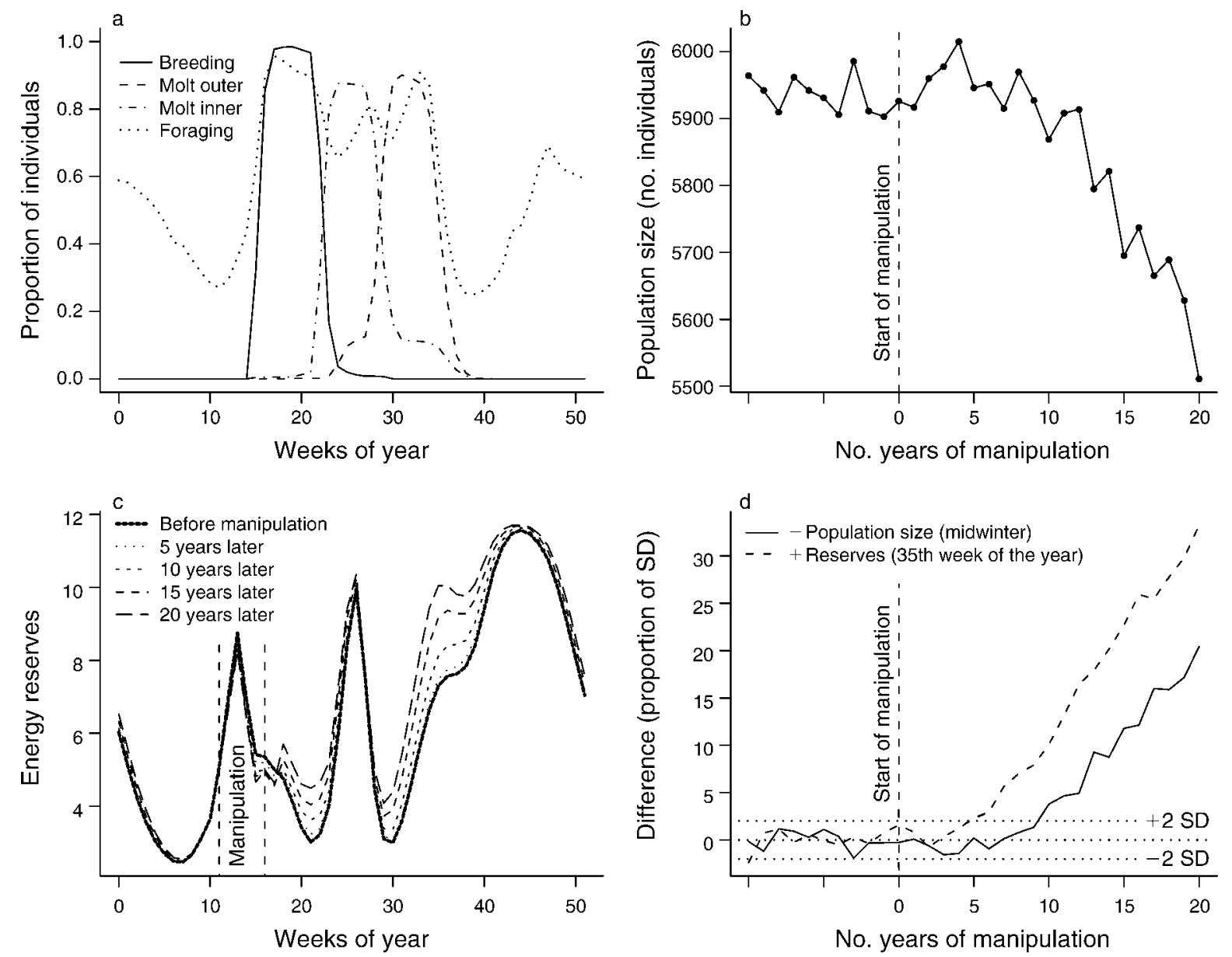

FIg. 1. The model of optimal molt. (a) The optimal annual routine. The figure shows the proportion of individuals following the given behavior, based on the individuals that survived the year (after Barta et al. 2006b). Molt outer and molt inner refers to, respectively, molting outer and molting inner primaries. (b) Effect of food reduction on population size (an illustration). The population is undisturbed until year 0 . After that, food availability between weeks 11 and 15 is reduced annually by $0.5 \%$ of the original value. (c) The effect of food reduction applied at the beginning of the breeding period on the course of energy reserves over the year. Energy reserves of the modeled birds are measured on a scale from 0 (starvation) to 12 (full energy reserves). The thick continuous line represents the mean over the years before the manipulation; the other lines show the means of energy reserves over the year, at five-year intervals 5-20 years after the start of food reduction. (d) Changes are shown as the proportion of the standard deviation of the given variable over the years before the manipulation. For ease of comparison, changes in population size are presented on a negative scale; thus, lines represent increases in energy reserves with decreases in population size (as the + and symbols indicate in the key).

those following the winter molt strategy were much more sensitive. Manipulations on the various sites had different effects. The populations following the summer molt strategy were most sensitive to environmental change on the wintering site, while populations that followed the winter molt strategy reacted nearly equally to manipulations on the breeding and wintering sites but did not respond as strongly to changes on the stopover sites (Fig. 2a). As in the previous example, increasing energy reserves were once again an early indication of the degradation of the environment (Fig. 2b).

\section{Discussion}

In this paper we have reviewed the methods currently used for predicting population processes and their response to environmental change (Table 1). In particular, we discussed different types of IBMs, approaches that focus on describing the actions of individuals and predicting population dynamics as an emergent phenomenon of simple rules that determine individual behavior. We recognize that many excellent, existing IBMs have generated useful insights into general ecological questions and specific applied problems (see recent reviews in DeAngelis and Mooij 2005, Sutherland 2006). Nevertheless, IBMs are not without limitations; we contend that several of those limitations can be overcome by the use of OAR models for population prediction.

One type of limitation of IBMs is derived from the method used to establish the behavioral rules that 


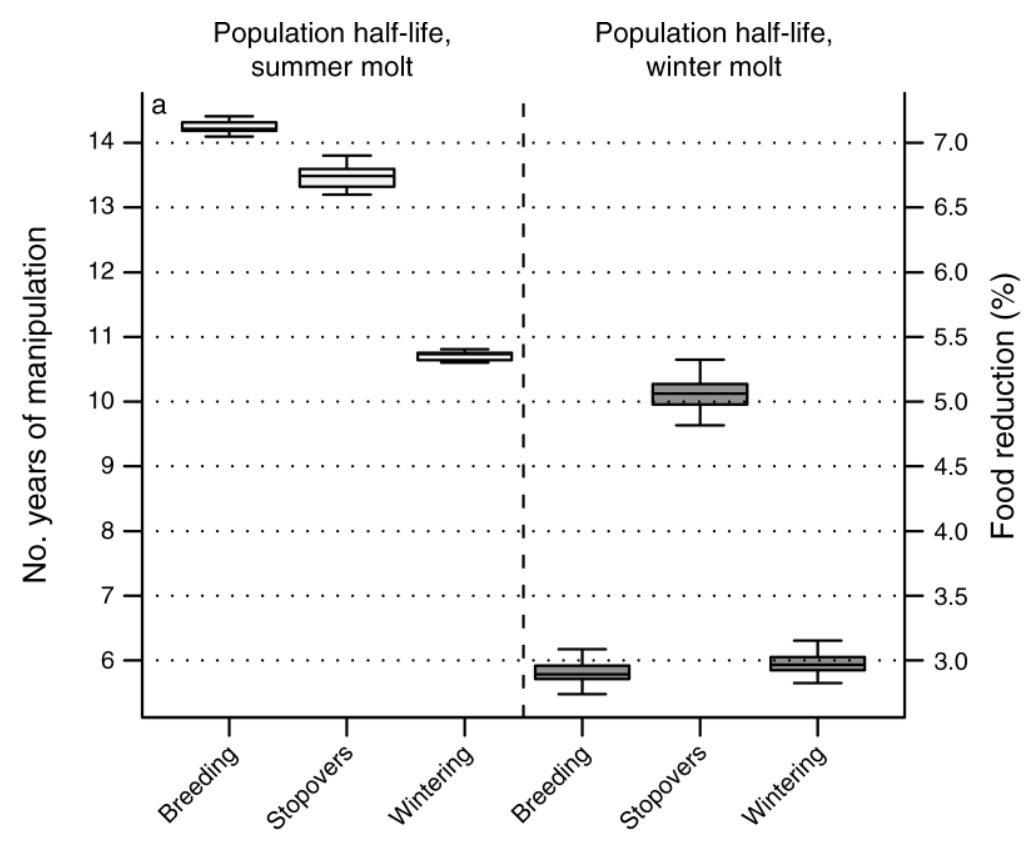

Site of manipulation

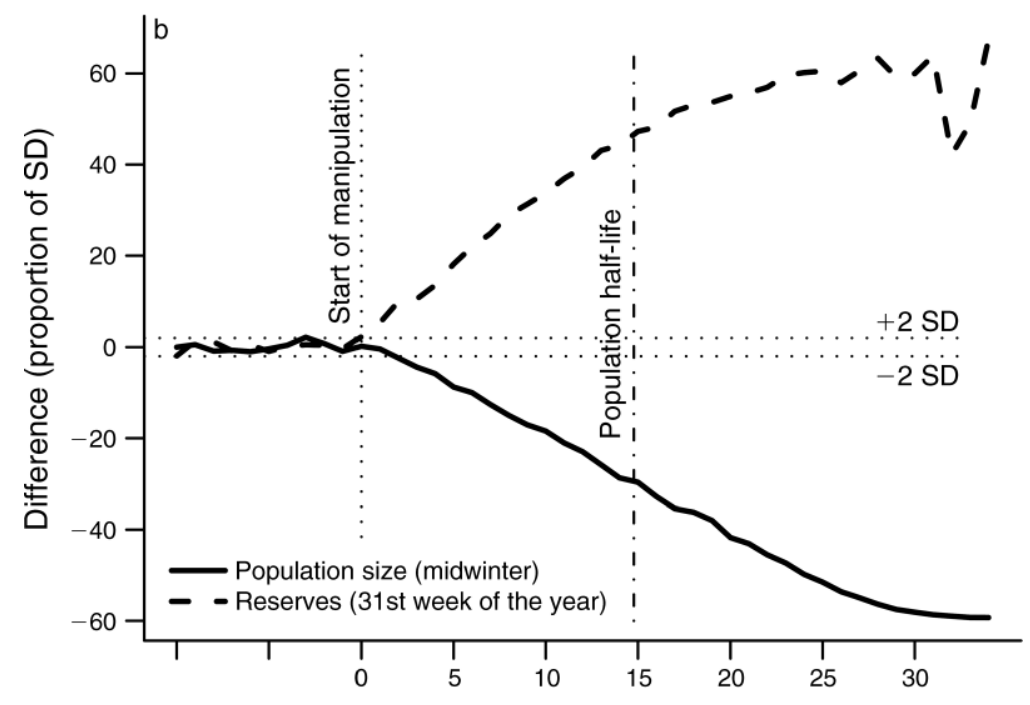

No. years of manipulation

FIG. 2. The model of optimal molt and migration. (a) Effects of food reduction on population size. The food reduction was applied from the 30th year at various sites along the migration route. We studied two populations: individuals of one population molt on the breeding site immediately after breeding (summer molt) while individuals of the other population molt on the wintering site after migration (winter molt). The half-life value is the time (years) elapsed until the population size halved. Boxplots represent the median, the interquartile range, and the maximum and minimum of half-life values from 10 repeats of the simulation. (b) Changes in population size and energy reserves resulting from food reduction on the stopover sites (summer molt). Changes are shown as a proportion of the standard deviation of the given variable over the years before the manipulation.

determine fates of individuals. Empirically derived rules can be determined relatively easily but do not allow for adaptive adjustments of individual behavior to changing conditions (since they lack any mechanistic underpinning for re-deriving the relevant probabilities). These methods can be suitable for exposing the processes operating within populations under observed conditions but should not be used for predictive modeling, because empirical probabilities cannot be expected to remain consistent under novel circumstances.

Optimality-based IBMs overcome many of the limitations of probabilistic rules by using behavioral rules that are likely to maximize the fitness of individuals. Short-term fitness proxies can be used in 
TABLE 1. Advantages and disadvantages of different modeling methods in the context of predictive population ecology.

\begin{tabular}{|c|c|c|c|}
\hline Modeling approach & Advantages & Disadvantages & Reference \\
\hline $\begin{array}{l}\text { Traditional age- or } \\
\text { stage-based } \\
\text { matrix models }\end{array}$ & $\begin{array}{l}\text { Relatively simple to construct. } \\
\text { Readily analyzed to provide } \\
\text { population characteristics such } \\
\text { as growth rate and stable age } \\
\text { structure. Standard methods } \\
\text { exist for sensitivity and } \\
\text { elasticity analyses. }\end{array}$ & $\begin{array}{l}\text { Cannot easily incorporate spatial } \\
\text { information or patterns of } \\
\text { relatedness. Inclusion of many } \\
\text { state variables leads to unwieldy } \\
\text { matrices. Transition } \\
\text { probabilities are typically } \\
\text { empirically derived (problems } \\
\text { are noted in this column). }\end{array}$ & Caswell (2001) \\
\hline $\begin{array}{l}\text { Individual-based models } \\
\text { (IBMs) with empirically } \\
\text { derived behavioral rules }\end{array}$ & $\begin{array}{l}\text { Well suited to exposing processes } \\
\text { operating in stable populations } \\
\text { and for determining sensitivity } \\
\text { of population parameters to } \\
\text { underlying behaviors (thus } \\
\text { guiding empirical research } \\
\text { efforts to understand the critical } \\
\text { behaviors). }\end{array}$ & $\begin{array}{l}\text { Empirical data on many behaviors } \\
\text { (especially, for example, the } \\
\text { fates of dispersers) can be very } \\
\text { difficult to collect. Measured } \\
\text { probabilities of behaviors or } \\
\text { state transitions cannot be } \\
\text { applied to novel conditions with } \\
\text { confidence, preventing their use } \\
\text { for predictive modeling. }\end{array}$ & $\begin{array}{l}\text { DeAngelis and Gross } \\
\text { (1992) }\end{array}$ \\
\hline $\begin{array}{l}\text { IBMs with optimality- } \\
\text { based rules }\end{array}$ & $\begin{array}{l}\text { As previously stated in this table. } \\
\text { Additionally, can be applied to } \\
\text { novel circumstances with } \\
\text { greater confidence as, in some } \\
\text { cases, they can capture the key } \\
\text { life-history decisions that will } \\
\text { dictate population dynamics in } \\
\text { novel conditions. }\end{array}$ & $\begin{array}{l}\text { Data hungry and, hence, difficult } \\
\text { to parameterize and validate. } \\
\text { Not well suited to incorporating } \\
\text { life-history trade-offs among } \\
\text { behavioral options and thus } \\
\text { limited to modeling scenarios } \\
\text { with relatively restricted } \\
\text { behavioral options. }\end{array}$ & Sutherland (1996) \\
\hline $\begin{array}{l}\text { Optimal annual routine } \\
\text { models }\end{array}$ & $\begin{array}{l}\text { Incorporate life-history trade-offs } \\
\text { and produce optimal behaviors } \\
\text { for full life-histories. Population } \\
\text { dynamics and fitness are } \\
\text { emergent phenomena. Fully } \\
\text { applicable to novel } \\
\text { circumstances. Provide } \\
\text { individual-level indicators that } \\
\text { can facilitate validation and } \\
\text { may act as sensitive indicators } \\
\text { of change. }\end{array}$ & $\begin{array}{l}\text { Computationally complex and } \\
\text { difficult to parameterize. } \\
\text { Require highly focused analysis } \\
\text { or risk overwhelming users with } \\
\text { data produced. }\end{array}$ & $\begin{array}{l}\text { Houston and } \\
\text { McNamara (1999) }\end{array}$ \\
\hline
\end{tabular}

some cases and have been shown to work in a number of tests of optimal behavior (e.g., Carlson 1983, Kacelnik 1984, Gill et al. 2001, Parker 2001). The main problem with these methods is that in some situations it is not certain that a short-term fitness proxy is also an appropriate indicator of fitness consequences in the longer term (Houston et al. 1988, Mangel and Clark 1988, Houston and McNamara 1999, Clark and Mangel 2000). It may also be difficult to incorporate life history trade-offs between different elements of fitness in the model. Long-term fitness projections are well suited to exposing processes operating in stable populations (e.g., Ridley et al. 2003). However, it is complicated to incorporate future choices into current projections and to consider feedback between estimates of fitness and future conditions; thus, these models require restrictive assumptions that limit their application to population prediction.

A significant advantage of the approach of OARs is that they can be used to evaluate the long-term fitness consequences of behavioral decisions, enabling predictions of the optimal behavior of individuals over their entire lifetime. This does not require direct estimation of the fitness consequences of actions; using the technique of dynamic programming, it is sufficient to define only the impacts of actions on the state of individuals. In this way, trade-offs and the effects of possible future choices and conditions on fitness are readily incorporated.

In OARs, individuals make behavioral decisions using an optimal strategy that depends on their state and some environmental factors, adjusting their behavior according to the changing internal and external circumstances. By simulating individual behavior in changing environments, it is possible to predict the consequences of those changes for population trends. A range of approaches could be used to make these predictions. In this paper, we illustrated an approach based on the assumption that the environment will change much more rapidly than evolution can act to alter behavioral strategies (especially likely given the speed of environmental change in recent times). In this case, the optimal state dependent strategy is determined once (for initial conditions) and then followed in spite of changing conditions. This "static strategies" approach means that individuals respond to changes in the environment only because of the effects that those changes have on their state (e.g., reduced food availability leads to reduced energy reserves, causing individuals to pursue strategies associated with lower reserves). Individual flexibility can be increased further by explicitly incorporating environ- 
mental variables (e.g., food availability) in the animals' state. Yet another approach (still within the framework of state-dependent optimal annual routines) would be to consider behavioral evolution as a response to the changing environment (McNamara and Houston 2008). In this case, optimal strategies are re-derived and updated to account for changing environmental circumstances. In reality, the situation might be intermediate between these extremes, with some lag between environmental change and behavioral adaptation. Determining the speed and consequences of behavioral responses to environmental change remains a major challenge for both empiricists and theoreticians (Barrett and Shluter 2008, Visser 2008). More generally, it can be relevant to analyze how ecological and evolutionary processes affect each other when constructing predictive population models. There has been growing interest in this relation recently (e.g., Thompson 1998, Hairston et al. 2005, Saccheri and Hanski 2006), and several approaches now link ecological and evolutionary dynamics in this way (e.g., Coulson et al. 2006, Pelletier et al. 2007).

In contrast to most optimality-based IBMs that make predictions only at the population (and, perhaps, behavioral) level, OAR models also provide individuallevel predictions. Although the models remain data hungry, their predictions have the potential to improve the efficiency and accuracy of model parameterization and testing. Moreover, since they predict not only population trends but the behavior and state of the individuals as well, OARs can be used as an effective and sensitive method for basing population monitoring on individual attributes.

To exploit the advantages of OAR models for conservation, further research is needed, both theoretical and empirical. Theoretical studies are required to determine the relationship between state variables and environmental factors, and to establish how sensitive these new methods are to the details of the models. Equally, empirical studies are necessary to test the assumptions and predictions of the models. Although we acknowledge that OARs do not offer a panacea for all the problems of other predictive population models, we believe (for a range of reasons detailed here) that OAR models do have clear potential for predictive population modeling. We urge collaborations between empiricists and theoreticians to explore that potential in applied contexts.

\section{ACKNOWLEDGMENTS}

We are very grateful to Ben Cairns for his valuable comments on the earlier versions of the manuscript. We also thank Tim Coulson and an anonymous reviewer for helping to improve the accuracy and intelligibility of the manuscript. Theoretical developments and modeling work were supported by OTKA (grants T046661, NF061143). P. A. Stephens was supported by the Natural Environment Research Council (grant number 2003/00616), Z. Barta was supported by the Öveges Fellowship, and by a BBSRC grant to A. I. Houston and J. M. McNamara.

\section{Literature Cited}

Akçakaya, H. R., M. A. Burgman, O. Kindvall, C. C. Wood, P. Sjögren-Gulve, J. S. Hatfield, and M. A. McCarthy. 2004. Species conservation and management. Oxford University Press, New York, New York, USA.

Arnold, W. 1990a. The evolution of marmot sociality: I. Why disperse late? Behavioral Ecology and Sociobiology 27:229237.

Arnold, W. 1990b. The evolution of marmot sociality: II. Costs and benefits of joint hibernation. Behavioral Ecology and Sociobiology 27:239-246.

Barrett, D. H., and D. Schluter. 2008. Adaptation from standing genetic variation. Trends in Ecology and Evolution 23:38-44.

Barta, Z., O. Feró, A. I. Houston, and J. M. McNamara. 2006a. Optimal immunity over the annual cycle in nonmigratory birds. Journal of Ornithology 147:31-31.

Barta, Z., A. I. Houston, J. M. McNamara, R. K. Welham, A. Hedenström, T. P. Weber, and O. Feró. 2006b. Annual routines of non-migratory birds: optimal moult strategies. Oikos 112:580-593.

Barta, Z., J. M. McNamara, A. I. Houston, T. P. Weber, A. Hedenström, and O. Feró. 2008. Optimal moult strategies in migratory birds. Philosophical Transactions of the Royal Society of London Series B 363:211-229.

Becker, P. H., C. C. Voigt, J. M. Arnold, and R. Nagel. 2006. A non-invasive technique to bleed incubating birds without trapping: a blood-sucking bug in a hollow egg. Journal of Ornithology 147:115-118.

Beissinger, S. R., and M. I. Westphal. 1998. On the use of demographic models of population viability in endangered species management. Journal of Wildlife Management 62: 821-841.

Bierzychudek, P. 1999. Looking backwards: assessing the projections of a transition matrix model. Ecological Applications 9:1278-1287.

Boyce, M. S. 1992. Population viability analysis. Annual Review of Ecology and Systematics 23:481-506.

Bradbury, R. B., R. J. H. Payne, J. D. Wilson, and J. R. Krebs. 2001. Predicting population responses to resource management. Trends in Ecology and Evolution 16:440-445.

Brook, B. W., M. A. Burgman, H. R. Akçakaya, J. J. O'Grady, and R. Frankham. 2002. Critiques of PVA ask the wrong questions: throwing the heuristic baby out with the numerical bath water. Conservation Biology 16:262-263.

Brook, B. W., J. J. O'Grady, A. P. Chapman, M. A. Burgman, H. R. Akçakaya, and R. Frankham. 2000. Predictive accuracy of population viability analysis in conservation biology. Nature 404:385-387.

Bunnell, D. B., and E. A. Marschall. 2003. Optimal energy allocation to ovaries after spawning. Evolutionary Ecology Research 5:439-457.

Carlson, A. 1983. Maximizing energy delivery to dependent young - field experiment with Red-Backed Shrikes (Lanius collurio). Journal of Animal Ecology 52:697-704.

Caswell, H. 2001. Matrix population models. Second edition. Sinauer Associates, Sunderland, Massachusetts, USA.

Clark, C. W., and M. Mangel. 2000. Dynamic state variable models in ecology: methods and applications. Oxford University Press, Oxford, UK.

Cote, S. D., and M. Festa-Bianchet. 2001. Birthdate, mass and survival in mountain goat kids: effects of maternal characteristics and forage quality. Oecologia 127:230-238.

Coulson, T., T. G. Benton, P. Lundberg, S. R. X. Dall, B. E. Kendall, and J.-M. Gaillard. 2006. Proceedings of the Royal Society of London B 273:547-555.

Coulson, T., G. M. Mace, E. Hudson, and H. P. Possingham. 2001. The use and abuse of population viability analysis. Trends in Ecology and Evolution 16:219-221. 
DeAngelis, D. L., and L. J. Gross. 1992. Individual-based models and approaches in ecology. Chapman and Hall, London, UK.

DeAngelis, D. L., and W. M. Mooij. 2005. Individual-based modeling of ecological and evolutionary processes. Annual Review of Ecology Evolution and Systematics 36:147-168.

Doherty, P. F., E. A. Marschall, and T. C. Grubb. 1999. Balancing conservation and economic gain: a dynamic programming approach. Ecological Economics 29:349-358.

Ellner, S. P., J. Fieberg, D. Ludwig, and C. Wilcox. 2002. Precision of population viability analysis. Conservation Biology 16:258-261.

Fauchald, P., T. Tveraa, C. Henaug, and N. Yoccoz. 2004. Adaptive regulation of body reserves in reindeer, Rangifer tarandus: a feeding experiment. Oikos 107:583-591.

Foster, M. S. 1975. The overlap of molting and breeding in some tropical birds. Condor 77:304-314.

Freckleton, R. P., P. A. Stephens, W. J. Sutherland, and A. R. Watkinson. 2004. Amelioration of biodiversity impacts of genetically modified crops: predicting transient versus longterm effects. Proceedings of the Royal Society of London B 271:325-331.

Fretwell, S. D., and J. H. J. Lucas. 1970. On territorial behaviour and other factors influencing habitat distribution in birds. Acta Biotheoretica 19:16-36.

Garvey, J. E., and E. A. Marschall. 2003. Understanding latitudinal trends in fish body size through models of optimal seasonal energy allocation. Canadian Journal of Fisheries and Aquatic Sciences 60:938-948.

Gill, J. A., W. J. Sutherland, and K. Norris. 2001. Depletion models can predict shorebird distribution at different spatial scales. Proceedings of the Royal Society of London B 268: 369-376.

Gilpin, M. E., and M. E. Soulé. 1986. Minimum viable populations: processes of species extinction. Pages 19-34 in M. E. Soule, editor. Conservation biology: the science of scarcity and diversity. Sinauer Associates, Sunderland, Massachusetts, USA.

Gosler, A. G., J. J. D. Greenwood, and C. Perrins. 1995. Predation risk and the cost of being fat. Nature 377:621-623.

Goss-Custard, J. D., and W. J. Sutherland. 1997. Individual behaviour, populations and conservation. Pages 373-395 in J. R. Krebs and N. B. Davies, editors. Behavioural ecology, an evolutionary approach. Fourth edition. Blackwell, Oxford, UK.

Goss-Custard, J. D., A. D. West, R. W. G. Caldow, S. E. A. L. D. Durell, S. McGrorty, and J. Urfi. 1996. An empirical optimality model to predict the intake rates of Oystercatchers Haematopus ostralegus feeding on mussels Mytilus edulis. Ardea 84A:199-214.

Green, W. C. H., and A. Rothstein. 1993. Persistent influences of birth date on dominance, growth and reproductive success in bison. Journal of Zoology 230:177-186.

Grimm, V. 1999. Ten years of individual-based modelling in ecology: what have we learned and what could we learn in the future? Ecological Modelling 115:129-148.

Grimm, V., et al. 2006. A standard protocol for describing individual-based and agent-based models. Ecological Modelling 198:115-126.

Grimm, V., N. Dorndorf, F. Frey-Roos, C. Wissel, T. Wyszomirski, and W. Arnold. 2003. Modelling the role of social behavior in the persistence of the alpine marmot Marmota marmota. Oikos 102:124-136.

Grimm, V., and S. F. Railsback. 2005. Individual-based modeling and ecology. Princeton University Press, Princeton, New Jersey, USA.

Hairston, N. G., S. P. Ellner, M. A. Geber, T. Yoshida, and J. A. Fox. 2005. Rapid evolution and the convergence of ecological and evolutionary time. Ecology Letters 8:11141127.
Hamilton, S., and H. Moller. 1995. Can PVA models using computer packages offer useful conservation advice? Sooty Shearwaters Puffinus griseus in New Zealand as a case study. Biological Conservation 73:107-117.

Hill, G. E. 1995. Ornamental traits as indicators of environmental health. BioScience 45:25-31.

Hole, D. G., M. J. Whittingham, R. B. Bradbury, G. Q. A. Anderson, P. L. M. Lee, J. D. Wilson, and J. R. Krebs. 2002. Widespread local house-sparrow extinctions - agricultural intensification is blamed for the plummeting populations of these birds. Nature 418:931-932.

Houston, A. I., C. W. Clark, J. M. McNamara, and M. Mangel. 1988. Dynamic models in behavioural and evolutionary ecology. Nature 332:29-34.

Houston, A. I., A. Kacelnik, and J. M. McNamara. 1982. Some learning rules for acquiring information. Pages 140-191 in D. J. McFarland, editor. Functional ontogeny. Pitman, London, USA.

Houston, A. I., and J. M. McNamara. 1997. Patch choice and population size. Evolutionary Ecology 11:703-722.

Houston, A. I., and J. M. McNamara. 1999. Models of adaptive behaviour: an approach based on state. Cambridge University Press, Cambridge, UK.

Huston, M., D. DeAngelis, and W. Post. 1988. New computer models unify ecological theory. BioScience 38:682-691.

Judson, O. P. 1994. The rise of the individual-based model in ecology. Trends in Ecology and Evolution 9:9-14.

Kacelnik, A. 1984. Central place foraging in Starlings (Sturnus vulgaris). 1. Patch residence time. Journal of Animal Ecology 53:283-299.

Lack, D. 1968. Ecological adaptations for breeding in birds. Chapman and Hall, London, UK.

Lank, D. B., and R. C. Ydenberg. 2003. Death and danger at migratory stopovers: problems with "predation risk." Journal of Avian Biology 34:225-228.

Lewis, S., D. Gremillet, F. Daunt, P. G. Ryan, R. J. M. Crawford, and S. Wanless. 2006. Using behavioural and state variables to identify proximate causes of population change in a seabird. Oecologia 147:606-614.

Łomnicki, A. 1999. Individual-based models and the individualbased approach to population ecology. Ecological Modelling 115:191-198.

Mangel, M., and C. W. Clark. 1988. Dynamic modeling in behavioral ecology. Princeton University Press, Princeton, New Jersey, USA.

Marra, P. P., K. A. Hobson, and R. T. Holmes. 1998. Linking winter and summer events in a migratory bird by using stable-carbon isotopes. Science 282:1884-1886.

Matsinos, Y. G., W. F. Wolff, and D. L. DeAngelis. 2002. Can individual-based models yield a better assessment of population variability? Pages 188-198 in S. Ferson and M. Burgman, editors. Quantitative methods for conservation biology. Springer, New York, New York, USA.

Maynard Smith, J. 1982. Evolution and the theory of games. Cambridge University Press, Cambridge, UK.

McNamara, J. M., Z. Barta, M. Wikelski, and A. I. Houston. In press. A theoretical investigation of the effect of latitude on avian life histories. American Naturalist.

McNamara, J. M., and A. I. Houston. 1980. The application of statistical decision theory to animal behaviour. Journal of Theoretical Biology 85:673-690.

McNamara, J. M., and A. I. Houston. 1987. Starvation and predation as factors limiting population size. Ecology 68: $1515-1519$.

McNamara, J. M., and A. I. Houston. 1996. State-dependent life histories. Nature 380:215-221.

McNamara, J. M., and A. I. Houston. 2008. Optimal annual routines: behaviour in the context of physiology and ecology. Philosophical Transactions of the Royal Society of London B 363:301-319. 
McNamara, J. M., J. N. Webb, E. J. Collins, T. Szekely, and A. I. Houston. 1997. A general technique for computing evolutionarily stable strategies based on errors in decision making. Journal of Theoretical Biology 189:211-225.

McNamara, J. M., R. K. Welham, and A. I. Houston. 1998. The timing of migration within the context of an annual routine. Journal of Avian Biology 29:416-423.

McNamara, J. M., R. K. Welham, A. I. Houston, S. Daan, and J. M. Tinbergen. 2004. The effects of background mortality on optimal reproduction in a seasonal environment. Theoretical Population Biology 65:361-372.

Newton, I. 2004. Population limitation in migrants. Ibis 146: 197-226.

Newton, I. 2006. Can conditions experienced during migration limit the population levels of birds? Journal of Ornithology 147:146-166.

Norris, D. R. 2005. Carry-over effects and habitat quality in migratory populations. Oikos 109:178-186.

Norris, K., and R. A. Stillman. 2002. Predicting the impact of environmental change. Pages 180-201 in K. Norris and D. J. Pain, editors. Conserving bird biodiversity. Cambridge University Press, Cambridge, UK.

Pap, T. L. 2002. Breeding time and sex-specific health status in the barn swallow (Hirundo rustica). Canadian Journal of Zoology 80:2090-2099.

Parker, G. A. 2001. Golden flies, sunlit meadows: a tribute to the yellow dungfly. Pages 3-26 in L. A. Dugatkin, editor. Model systems in behavioural ecology: integrating conceptual, theoretical, and empirical approaches. Princeton University Press, Princeton, New Jersey, USA.

Peach, W. J., G. M. Siriwardena, and R. D. Gregory. 1999. Long-term changes in over-winter survival rates explain the decline of reed buntings Emberiza schoeniclus in Britain. Journal of Applied Ecology 36:798-811.

Pelletier, F., T. Clutton-Brock, J. Pemberton, S. Tuljapurkar, and T. Coulson. 2007. The evolutionary demography of ecological change: linking trait variation and population growth. Science 315:1571-1574.

Pettifor, R. A., R. W. G. Caldow, J. M. Rowcliffe, J. D. GossCustard, J. M. Black, K. H. Hodder, A. I. Houston, A. Lang, and J. Webb. 2000. Spatially explicit, individual-based, behavioural models of the annual cycle of two migratory goose populations. Journal of Applied Ecology 37:103-135.

Pierce, G. J., and J. G. Ollason. 1987. Eight reasons why optimal foraging theory is a complete waste of time. Oikos 49:111-118.

Piersma, T., J. Vangils, P. Degoeij, and J. Vandermeer. 1995. Holling's functional-response model as a tool to link the food-finding mechanism of a probing shorebird with its spatial distribution. Journal of Animal Ecology 64:493-504.

Reed, J. M., D. D. Murphy, and P. F. Brussard. 1998. Efficacy of population viability analysis. Wildlife Society Bulletin 26: 244-251.

Ridley, J., J. Komdeur, and W. J. Sutherland. 2003. Population regulation in group-living birds: predictive models of the Seychelles warbler. Journal of Animal Ecology 72:588-598.

Rogers, C. M., V. Nolan, and E. D. Ketterson. 1994. Winter fattening in the dark-eyed junco: plasticity and possible interaction with migration trade-offs. Oecologia 97:526-532.

Saccheri, I., and I. Hanski. 2006. Natural selection and population dynamics. Trends in Ecology and Evolution 21: 341-347.

Sadedin, S., and M. J. Littlejohn. 2003. A spatially explicit individual-based model of reinforcement in hybrid zones. Evolution 57:962-970.

Shaffer, M. L. 1981. Minimum population sizes for species conservation. BioScience 31:131-134.

Shugart, H. H., T. M. Smith, and W. M. Post. 1992. The potential for application of individual-based simulation models for assessing the effects of global change. Annual Review of Ecology and Systematics 23:15-38.

Stearns, S. C. 1992. The evolution of life histories. Oxford University Press, Oxford, UK.

Stearns, S. C., and P. Schmid-Hempel. 1987. Evolutionary insights should not be wasted. Oikos 49:118-125.

Stephens, D. W., and J. R. Krebs. 1986. Foraging theory. Princeton University Press, Princeton, New Jersey, USA.

Stephens, P. A., R. P. Freckleton, A. R. Watkinson, and W. J. Sutherland. 2003. Predicting the response of farmland bird populations to changing food supplies. Journal of Applied Ecology 40:970-983.

Stephens, P. A., F. Frey-Roos, W. Arnold, and W. J. Sutherland. 2002a. Model complexity and population predictions. The alpine marmot as a case study. Journal of Animal Ecology 71:343-361.

Stephens, P. A., F. Frey-Roos, W. Arnold, and W. J. Sutherland. 2002b. Sustainable exploitation of social species: a test and comparison of models. Journal of Applied Ecology 39:629-642.

Stephens, P. A., A. F. Russell, A. J. Young, W. J. Sutherland, and T. H. Clutton-Brock. 2005. Dispersal, eviction, and conflict in meerkats (Suricata suricatta): an evolutionarily stable strategy model. American Naturalist 165:120-135.

Stillman, R. A., J. D. Goss-Custard, A. D. West, S. E. A. L. D. Durell, R. W. G. Caldow, S. McGrorty, and R. T. Clarke. 2000. Predicting mortality in novel environments: tests and sensitivity of a behaviour-based model. Journal of Applied Ecology 37:564-588.

Stillman, R. A., J. D. Goss-Custard, A. D. West, S. E. A. L. D. Durell, S. McGrorty, R. W. G. Caldow, K. J. Norris, I. G. Johnstone, B. J. Ens, J. Van der Meer, and P. Triplet. 2001. Predicting shorebird mortality and population size under different regimes of shellfishery management. Journal of Applied Ecology 38:857-868.

Stillman, R. A., A. E. Poole, J. D. Goss-Custard, R. W. G. Caldow, M. G. Yates, and P. Triplet. 2002. Predicting the strength of interference more quickly using behaviour-based models. Journal of Animal Ecology 71:532-541.

Stillman, R. A., and V. L. Simmons. 2006. Predicting the functional response of a farmland bird. Functional Ecology 20:723-730.

Stillman, R. A., A. D. West, J. D. Goss-Custard, R. W. G. Caldow, S. McGrorty, S. E. A. L. D. Durell, M. G. Yates, P. W. Atkinson, N. A. Clark, M. C. Bell, P. J. Dare, and M. Mander. 2003. An individual behaviour-based model can predict shorebird mortality using routinely collected shellfishery data. Journal of Applied Ecology 40:1090-1101.

Sutherland, W. J. 1996. From individual behaviour to population ecology. Oxford University Press, Oxford, UK.

Sutherland, W. J. 1998. The importance of behavioural studies in conservation biology. Animal Behaviour 56:801-809.

Sutherland, W. J. 2006. Predicting the ecological consequences of environmental change: a review of the methods. Journal of Applied Ecology 43:599-616.

Sutherland, W. J., and G. A. Allport. 1994. A spatial depletion model of the interaction between bean geese and wigeon with the consequences for habitat management. Journal of Animal Ecology 63:51-59.

Sutherland, W. J., and K. Norris. 2002. Behavioural models of population growth rates: implications for conservation and prediction. Philosophical Transactions of the Royal Society of London B 357:1273-1284.

Sutherland, W. J., and A. R. Watkinson. 2001. Policy making within ecological uncertainty: lessons from badgers and GM crops. Trends in Ecology and Evolution 16:261-263.

Szekely, T., and I. C. Cuthill. 1999. Brood desertion in Kentish plover: the value of parental care. Behavioral Ecology 10: 191-197. 
Thompson, J. N. 1998. Rapid evolution as an ecological process. Trends in Ecology and Evolution 13:329-332.

Visser, M. E. 2008. Keeping up with a warmer world; assessing the rate of adaptation to climate change. Philosophical Transactions of the Royal Society of London B 275:649-659.

Webster, M. S., and P. P. Marra. 2005. The importance of understanding migratory connectivity and seasonal interactions. Pages 199-209 in R. Greenberg and P. P. Marra, editors. Birds of two worlds: the ecology and evolution of migration. Johns Hopkins University Press, Baltimore, Maryland, USA.
White, P. C. L., N. V. Jennings, A. R. Renwick, and N. H. L. Barker. 2005. Questionnaires in ecology: a review of past use and recommendations for best practice. Journal of Applied Ecology 42:421-430.

Witter, M. S., and I. C. Cuthill. 1993. The ecological costs of avian fat storage. Philosophical Transactions of the Royal Society of London B 340:73-92.

Witter, M. S., J. P. Swaddle, and I. C. Cuthill. 1995. Periodic food availability and strategic regulation of body mass in the European starling, Sturnus vulgaris. Functional Ecology 9: $568-574$ 